\title{
Criptorquidectomia em coelhos: modelo experimental para tratamento laparoscópico
}

\author{
Rabbit cryptorchidectomy: experimental model to laparoscopic \\ trainning
}

\section{Carlos Afonso de Castro Beck ${ }^{1}$ Ney Luis Pippi ${ }^{2}$ Maurício Veloso Brun ${ }^{3}$ Marshal Costa Leme ${ }^{3}$ Emerson Antônio Contesini ${ }^{4}$ Rafael Stedile $^{5}$}

\section{RESUMO}

O criptorquidismo é considerado a afecção testicular congênita mais freqüente nos animais domésticos, tendo como indicação terapêutica a exérese testicular. No presente estudo, os autores desenvolveram um modelo de criptorquidismo unilateral em coelhos com o objetivo de testar sua viabilidade para o treinamento laparoscópico diagnóstico e cirúrgico, que permitisse extrapolar seus resultados para as pequenas espécies domésticas. A preparação do modelo foi de fácil execução e viabilizou de maneira satisfatória o treinamento laparoscópico, simulando a situação clínica encontrada nos pequenos animais.

Palavras-chave: cirurgia, videolaparoscopia, testículo.

\section{ABSTRACT}

Cryptorchidism is considered the congenital testicuiar disease most frequent in domestic animals, having the orchiectomy as therapeutical indication. In the present study, an unilateral cryptorchidism model in rabbit was developed, with the aim to test the viability for practicing laparoscopic diagnoses and surgery. The model preparation was easy to perform permiting the development of laparoscopic skills, simulating clinical situation in small animal practice.
Key words: cryptorchidism, rabbits, laparoscopic tranning.

\section{INTRODUÇÃO}

A não migração de um ou ambos os testículos até o escroto é denominada de criptorquidismo, sendo este a afecção testicular congênita mais comum nos animais domésticos (BOOTHE, 1998). O testículo ectópico pode ter posição pré-escrotal, inguinal ou abdominal e sua ocorrência pode ser uni ou bilateral (SW1NDLE, 1988; BOOTHE, 1998). O criptorquidismo unilateral é mais freqüente quando comparado ao bilateral, e o testículo direito de ocorrência maior (REIF \& BRODEY, 1969). Na Medicina Veterinária, não se justifica a tentativa de reposição do testículo no escroto ou orquipexia, pois devido à natureza hereditária do criptorquidismo, a permanência testicular possibilita a perpetuação do defeito (BURKE \& REYNOLDS 1983), além do alto risco de desenvolvimento de neoplasias nesses testículos ou em seus contralaterais (ROMAGNOLI, 1991; BOOTIIE,

\footnotetext{
${ }^{1}$ Médico Veterinário, Mestre, Professor Assistente do Departamento de Medicina Animal da Universidade Federal do Rio Grande do Sul (UFRGS). Av. Bento Gonçalves, 9090, 91540-000, Porto Alegre, RS, Brasil. Doutorando do Programa de Pós-graduação da Universidade Federal de Santa Maria (UFSM). E-mail: afonso@orion.ufrgs.br. Autor para correspondência.

${ }^{2}$ Médico Veterinário, PhD, Professor Titular, Departamento de Clínica de Pequenos Animais, UFSM.

${ }^{3}$ Médico Veterinário, Mestre, Doutorando do Programa de Pós-graduação da UFSM.

${ }^{4}$ Médico Veterinário, Mestre, Professor Assistente do Departamento de Medicina Animal da Universide Federal de Mato Grosso, (UFMT), Doutorando do Programa de Pós-graduação da UFSM.

${ }^{5}$ Aluno do Curso de Graduação em Medicina Veterinária da UFSM. Bolsista de Iniciação Científica CNPq.
} 
1998). A técnica cirúrgica para remoção de um testículo criptorquídico varia conforme a sua localização, sendo os de localização abdominal abordados, normalmente, por laparotomia mediana ventral (BOOTIIE, 1998).

Segundo FREEMAN (1998), a cirurgia endoscópica é um procedimento minimamente invasivo, no qual se utiliza um endoscópio com a finalidade de se visibilizarem diferentes órgãos ou cavidades. A autora caracteriza a laparoscopia como o acesso cirúrgico endoscópico ao abdome e destaca a importância do treinamento prévio, como etapa fundamental para a equipe cirúrgica antes de se realizarem cirurgias em casos clínicos. DENT (1992) ressalta a etapa de treinamento em animais de experimentação como essencial na formação dos cirurgiões que utilizarão a técnica laparoscópica. BECKER (1992) considera o treinamento em modelo animal muito eficaz, pois este, além de simular a situação clínica, permite a repetição dos procedimentos e possibilita aos membros da equipe cirúrgica a condição de participar do ato como cirurgião, primeiro assistente ou operador da câmara. BECK et al. (1996), FRIEDMAN \& PACE(1996) e FRIED et al. (1999) realizaram pesquisas que avaliaram o desempenho do cirurgião após participação em cursos de treinamento laparoscópico. Nesses estudos, concluíram que o treinamento possibilitou o aprimoramento dos cirurgiões em cirurgias endoscópicas.

$\mathrm{Na}$ Medicina, estudos têm mostrado a aplicação da técnica laparoscópica tanto no diagnóstico de testículos não palpáveis (FROELLNG et al., 1994; GHEILER et al., 1997), como, em alguns casos, em sua remoção cirúrgica (ANDZE et al.,1990; FROEL1NG et al., 1994: DE LA TORRE et al., 1995). Na Medicina Veterinária, apesar do número ainda reduzido, estudos têm descrito a técnica laparoscópica como acesso a casos de criptorquidismo em eqüinos (DAVIS, 1997; HENDRICKSON \& WILSON, 1997; FISCHER \& VACHON, 1998) e em cães (GOMEZ et al., 1996; FREEMAN \& HENDRICKSON, 1998; PENA et al., 1998). FREEMAN \& HENIDRICKSON (1998), consideraram a laparoscopia um meio excelente para estabelecer diagnóstico definitivo para os casos de testículos não-palpáveis em cães e em eqüinos, além de permitir a terapia cirúrgica para esses casos.

Trabalhos têm sido realizados na produção de modelos experimentais de criptorquidismo com o objetivo de avaliar as alterações reprodutivas geradas em conseqüência da posição ectópica dos testículos, ou de sua posterior reposição no interior do escroto, tanto em ratos (KOGAN et al., 1987; QUINN et al., 1991; SHONO \& SUITA, 1995), como em coelhos (SWINDLE, 1988; PRYOR et al., 1989).
Objetivou-se, com este trabalho, desenvolver um modelo de criptorquidismo abdominal unilateral em coelhos, avaliando sua viabilidade para o treinamento laparoscópico em medicina veterinária tanto para o diagnóstico como para a terapia cirúrgica de exérese testicular.

\section{MATERIAL E MÉTODOS}

O estudo foi desenvolvido no laboratório de Cirurgia Experimental da Universidade Federal de Santa Maria, utilizando seis coelhos, adultos, machos, da raça Nova Zelândia. Na primeira etapa do experimento, os animais foram submetidos a procedimento cirúrgico para indução do criptorquidismo unilateral.

O pré-operatório dos animais constou de jejum alimentar de seis horas, sendo realizada tricotomia da região abdominal ventral e superlicie escrotal. A anestesia foi realizada através da associação de cetamina $\left(12,5 \mathrm{mg} \mathrm{kg}^{-1}\right)$ e xilazina $\left(5 \mathrm{mg} \mathrm{kg}^{-1}\right)$ por via intramuscular, acrescida da administração local infiltrativa de lidocaína $2 \%\left(7 \mathrm{mg} \mathrm{kg}^{-1}\right)$ Como terapia antibiótica profilática, foi administrada ampicilina sódica $(20 \mathrm{mg} / \mathrm{kg})$, via intravenosa. Após o posicionamento dos coelhos em decúbito dorsal, foi promovida a antisepsia que observou a seqüência de álcool-iodo-álcool em toda a extensão da região cutânea tricotomizada. A incisão de pele e tecido subcutâneo foi realizada sobre a região do anel inguinal direito, em sentido oblíquo à linha alba e medindo aproximadamente $3 \mathrm{~cm}$ de extensão. Por meio de tração realizada no testículo direito sobre o escroto, o cordão testicular foi localizado e isolado, sendo a seguir identificado o anel inguinal externo, no ponto de emergência do cordão testicular (Figura $1 \mathrm{~A}$ ).

Com leve compressão sobre o testículo direito, foi possível introduzi-lo no interior da cavidade abdominal através do canal inguinal, mantendo-o nessa posição pela compressão digital do anel inguinal externo. Utilizando fio mononáilon n.3-0, foi realizado o fechamento do anel inguinal externo, por meio de duas suturas interrompidas em cruz (Figura 1B). O tecido subcutâneo foi aproximado através de sutura contínua simples e a pele suturada com pontos isolados simples. Ambas as suturas foram realizadas com mononáilon n.3-0 e os pontos de pele foram removidos sete dias após a cirurgia. Os animais foram mantidos por período de cinco semanas em alojamentos individuais, recebendo ração comercial balanceada e água ad libitum.

Na segunda etapa do experimento, realizada quatro semanas após a indução, os animais foram submetidos à criptorquidectomia laparoscópica, com a 


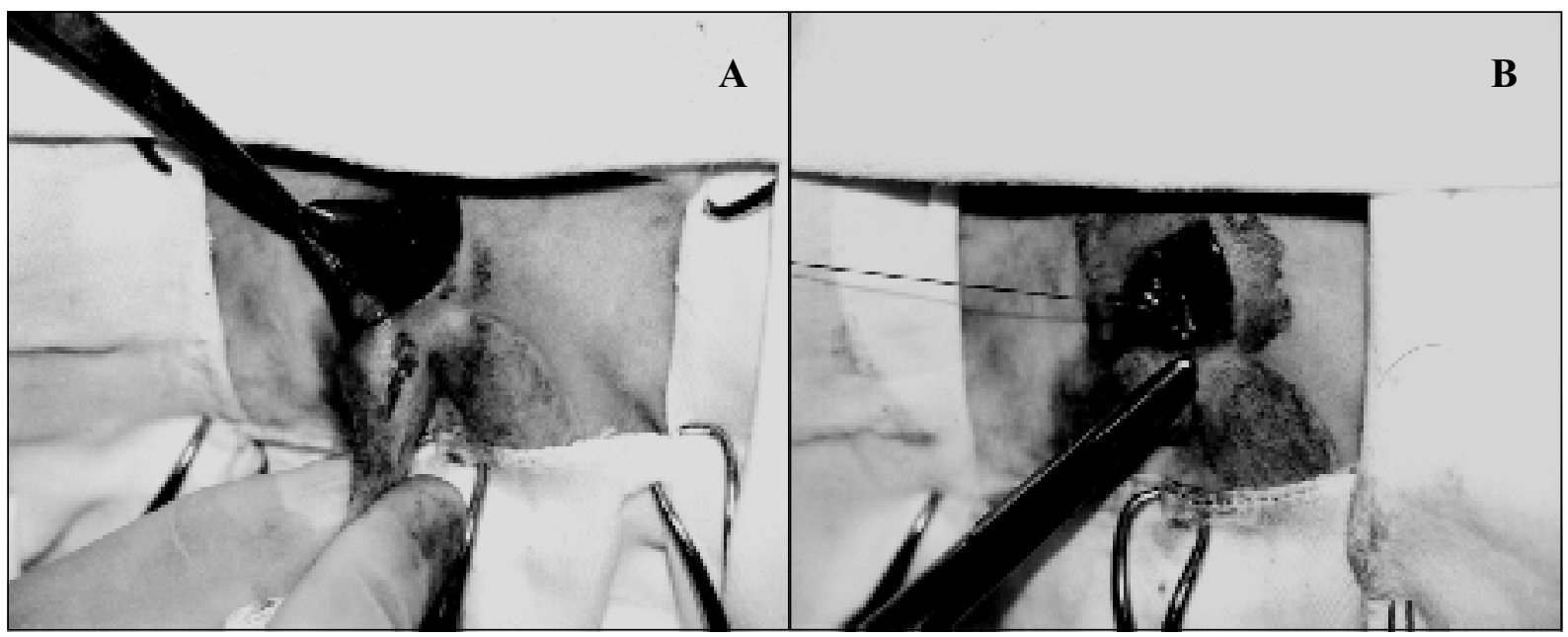

Figura 1 - Localização e isolamento do cordão testicular com identificação do anel inguinal externo em coelhos (A). Fechamento do anel inguinal externo através de sutura interrompida em cruz (B).

utilização de anestesia volátil com halotano, oferecido em oxigênio a $100 \%$, através de máscara facial adaptada ao sistema duplo $\mathrm{T}$ de Baraka. O procedimento cirúrgico teve início após o posicionamento dos animais em decúbito dorsal e realização da anti-sepsia. Com a utilização de duas pinças Backhaus sobre a região da cicatriz umbilical, a pele, tecido subcutâneo e músculo reto abdominal foram elevados. Após, promoveu-se a inserção da agulha de Veress em ângulo de $90^{\circ}$, em relação à linha alba. Uma seringa contendo solução de Ringer lactato foi adaptada ao canhão da agulha, com a finalidade de verificar a facilidade do fluxo para o interior da cavidade abdominal, certificando-se de sua correta localização. A seguir, a mangueira de silicone do insuflador de dióxido de carbono foi adaptada à agulha de Veress e a cavidade abdominal insuflada até atingir a pressão de $11 \mathrm{mmHg}$, sendo essa mantida em todo o procedimento. Durante a realização do pneumoperitônio, a parede abdominal foi percutida manualmente, para comprovar a distribuição homogênea do gás. Após alcançar a pressão desejada, a agulha de Veress foi removida.

Utilizando bisturi, foi realizada uma incisão de pele, de aproximadamente $1 \mathrm{~cm}$ de extensão, cerca de $5 \mathrm{~cm}$ cranial à cicatriz umbilical, sobre a linha alba. Através desta incisão, foi introduzido o primeiro trocarte, com diâmetro de $10 \mathrm{~mm}$. Tanto a inserção da agulha de Veress, como do primeiro trocarte, foram realizadas com ausência de visão direta. Através da cânula do primeiro trocarte, foi inserido um endoscópio rígido de $10 \mathrm{~mm}$ de diâmetro e ângulo de visão de zero grau, conectado a uma microcâmera e um cabo de luz de fibra óptica, sendo iniciada a exploração da cavidade abdominal. A exploração abdominal objetivou identificar, inicialmente, possíveis alterações em diferentes estruturas desta cavidade, bem como lesões provocadas pela inserção da agulha de Veress ou do primeiro trocarte. A seguir, foi feita a visibilização da região pélvica direita, sendo esse momento de observação considerado como diagnóstico laparoscópico do criptorquidismo. Tal manobra foi caracterizada como positiva quando o testículo ectópico foi identificado.

Sob visão direta do endoscópio, foi eleito o sítio de punção do segundo (10mm), e do terceiro trocartes $(5 \mathrm{~mm})$, respectivamente à direita e à esquerda do primeiro. Os sítios de punção dos três trocartes sofreram pequenas variações, conforme as dimensões e características anatômicas dos animais, mas guardaram sempre a disposição triangular (Figura 2). Com a utilização de uma pinça de Kelly no terceiro trocarte e uma tesoura no segundo, foi realizada a dissecação cuidadosa do cordão testicular, ducto deferente e músculo cremaster interno. A seguir, com a utilização de um aplicador de clipe de titânio, foi promovido o grampeamento dos vasos testiculares em dois pontos, sendo o primeiro proximal e o segundo distal ao ponto de secção desses. A mesma manobra foi realizada no ducto deferente. Após a aplicação dos clipes e secção das estruturas relacionadas, o testículo foi totalmente liberado no interior da cavidade abdominal. Através de um redutor adaptado ao segundo trocarte, foi introduzido um saco para remoção de vísceras, previamente enrolado em uma pinça de Kelly. O saco foi cuidadosamente desenrolado no interior da cavidade e o testículo introduzido em seu interior. Pela tração do fio em sua abertura, o saco foi obliterado. $\mathrm{O}$ segundo trocarte foi removido e pela sua abertura o 


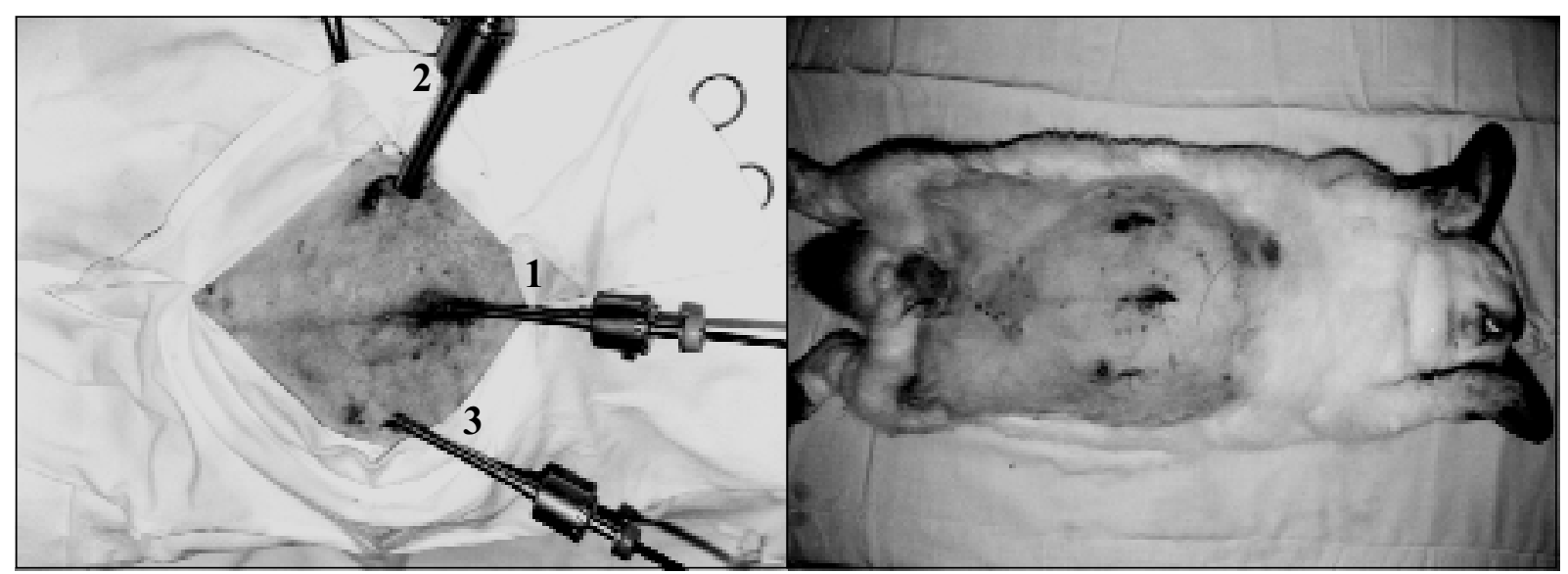

Figura 2 - Localização e numeração dos trocartes utilizados nos coelhos, destacando a distribuição triangular dos mesmos, com o endoscópio posicionado ao centro.

saco foi extraído da cavidade, sem a necessidade de se ampliar a incisão na parede abdominal.

Ao final, a cavidade foi novamente explorada, o pneumoperitônio gradualmente desfeito e os trocartes removidos. As incisões realizadas para a adaptação dos trocartes sofreram apenas sutura de tecido subcutâneo e pele, em pontos isolados simples, com fio mononáilon n.3-0. O testículo contralateral de todos os coelhos foi removido por técnica convencional, através da abertura do escroto e posterior ligadura e secção do cordão testicular. Ambos os testículos foram avaliados quanto às suas características macroscópicas, com o objetivo de verificar alterações em sua consistência e dimensão, decorrentes do período de cinco semanas, em que permaneceram na cavidade abdominal.

Para avaliação do procedimento laparoscópico, foram registrados os diferentes intervalos de tempo que compuseram as etapas cirúrgicas. O tempo cirúrgico total foi considerado desde a realização da primeira incisão de pele, até o último ponto cutâneo realizado, O tempo de pneumoperitônio foi estabelecido como o período do início da insuflação com $\mathrm{CO}_{2}$, até a remoção completa do gás, estando relacionado, diretamente, ao tempo de procedimento laparoscópico propriamente dito. $\mathrm{O}$ tempo de liberação testicular foi considerado o período entre o início da insuflação e a completa ligadura e liberação do testículo na cavidade e, por fim, o tempo de ensacamento, período compreendido entre a introdução do saco na cavidade, até a manobra de introdução do testículo no interior deste.

\section{RESULTADOS}

A técnica operatória utilizada para o preparo de modelo de criptorquidismo unilateral em coelhos mostrou-se efetiva e de fácil execução, com tempo cirúrgico que variou de 14 a 38 minutos e tempo médio de 24,6 minutos. Nenhum dos testículos, posicionado na cavidade abdominal, retornou ao interior do escroto. A simples compressão do testículo no fundo do escroto, proporcionou sua fácil migração ao abdome, através do canal inguinal e a aplicação de duas suturas interrompidas em cruz, garantiu o fechamento do anel inguinal externo, impedindo o reposicionamento escrotal do testículo. Dois coelhos removeram a sutura de pele no terceiro dia de pós-operatório, sendo a ferida cirúrgica parcialmente aberta e a cicatrização ocorrida por segunda intenção. Nos coelhos restantes, a sutura de pele foi removida ou desprendeu-se espontaneamente entre o quarto e o sétimo dia de pósoperatório, porém a cicatrização mostrou-se efetiva nesses animais. Durante o intervalo de cinco semanas entre os procedimentos cirúrgicos, nenhum dos coelhos evidenciou sinal de dor ou desconforto, mantendo o consumo normal de ração e água.

A segunda etapa do experimento, caracterizada pela identificação e remoção do testículo ectópico, através de acesso laparoscópico, igualmente mostrou-se efetiva. Nos seis coelhos, a técnica operatória utilizada permitiu a visibilização do testículo abdominal somente com a introdução do endoscópio e exploração abdominal, confirmando o diagnóstico positivo de criptorquidismo.

$\mathrm{O}$ equipamento e o instrumental cirúrgico utilizados mostraram-se apropriados para a realização dos procedimentos. Os tempos cirúrgicos para os seis coelhos, nas diferentes etapas dos procedimentos, encontram-se relacionados na tabela 1. Durante as manobras de introdução da agulha de Veress e do primeiro trocarte, não ocorreu punção acidental de vísceras abdominais nos animais. A utilização de três 
Tabela 1 - Dados referentes ao tempo de cirurgia total, tempo de pneumoperitônio, tempo de liberação testicular, tempo de ensacamento testicular e tempos médios dos coelhos submetidos à criptorquidectomia laparoscópica.

\begin{tabular}{lcccc}
\hline COELHO & $\begin{array}{c}\text { Tempo } \\
\text { cirúrgico total }\end{array}$ & $\begin{array}{c}\text { Tempo } \\
\text { pneumoperitônio }\end{array}$ & $\begin{array}{c}\text { Tempo liberação } \\
\text { testicular }\end{array}$ & $\begin{array}{c}\text { Tempo de } \\
\text { ensacamento }\end{array}$ \\
\hline 1 & 46 & 37 & 25 & 9 \\
2 & 42 & 34 & 22 & 7 \\
3 & 39 & 28 & 16 & 4 \\
4 & 37 & 23 & 11 & 5 \\
5 & 36 & 19 & 13 & 3 \\
6 & 33 & 20 & 13 & 1 \\
MÉDIA & 38,83 & 26,83 & 16,66 & 4,83 \\
\hline
\end{tabular}

OBS: Os valores de tempo estão registrados em minutos.

trocartes, um para o endoscópio e dois para a utilização de instrumentos laparoscópicos, bem como a localização dos mesmos, permitiu, além de adequada visibilização, perfeita mobilidade dos intrumentos nas diferentes etapas cirúrgicas. O pneumoperitônio, utilizando pressão constante de $11 \mathrm{mmHg}$, proporcionou excelente espaço de trabalho no interior da cavidade abdominal.

Em três coelhos foram utilizados três clipes de titânio para a realização da ligadura dos vasos do plexo pampiniforme e ducto deferente. Nos outros três animais, foram aplicados apenas dois clipes, não ocorrendo hemorragia em nenhum deles.

Dois coelhos apresentaram discreto enfisema subcutâneo no pós-operatório, tendo esse desaparecido espontaneamente ao final de 24 horas. Todos os testículos contidos na cavidade abdominal evidenciaram hipotrofia, após as cinco semanas.

\section{DISCUSSÃO}

A técnica operatória utilizada para o preparo do modelo de criptorquidismo foi semelhante à realizada por PRYOR et al. (1989), sem a necessidade da abertura da cavidade abdominal, utilizada por SWINDLE (1988). O acesso direto ao anel inguinal externo possibilitou a realização do procedimento em um tempo médio de 24,6 minutos, evidenciando sua fácil exequibilidade. A permanência do testículo na cavidade abdominal nos seis coelhos, após o fechamento do anel inguinal externo, durante o período de cinco semanas, indica a viabilidade do modelo de criptorquidismo nessa espécie.

A ocorrência de deiscência dos pontos de pele, em dois coelhos no segundo dia de pósoperatório, foi provocada por automutilação. Esse fato revelou que a sutura com pontos isolados na pele, com a permanência do material de sutura exteriorizado, deve ser evitada em coelhos, podendo ser testada a sutura intradérmica em futuros trabalhos.
No momento da exploração visual do abdome, realizada com a introdução do endoscópio, foi possível identificar o testículo criptorquidico nos seis coelhos, o que não somente confirmou a viabilidade do modelo experimental, como ressalta a importância do acesso laparoscópico no diagnóstico desta afecção cirúrgica, característica destacada por WILDT et al. (1977), FREEMAN \& HENDRICKSON (1998) e BRUN \& BECK (1999).

O posicionamento do coelho em decúbito dorsal e a utilização de três trocartes, sendo o do centro para inserção do endoscópio rígido e os dois restantes localizados lateralmente ao primeiro, em distribuição triangular, correspondem às etapas descritas por FREEMAN \& HENDRICKSON (1998) e GOMEZ et al. (1996), que relataram o procedimento em cães. O pneumoperitônio realizado com pressão constante de $11 \mathrm{mmHg}$ foi inferior aos valores de 12 a $14 \mathrm{mmHg}$, recomendados por FREEMAN \& HIENDRICKSON (1998) para este procedimento em cães. A pressão utilizada, no entanto, permitiu excelente visibilização da cavidade e espaço para manipulação do instrumental cirúrgico, reduzindo os riscos decorrentes de pressões superiores.

A punção acidental do baço e a ocorrência de enfisema subcutâneo, decorrentes, respectivamente, da introdução da agulha de Veress e da insuflação de $\mathrm{CO}_{2}$ através dessa, foram relacionadas por GILROY \& ANSON (1987), como complicações que podem ocorrer em cães, porém tais intercorrências não foram verificadas no presente estudo. $\mathrm{O}$ enfisema de subcutâneo ocorrido em dois coelhos, decorreu de extravasamento de $\mathrm{CO}_{2}$ ao redor dos trocartes. Este achado concorda com as considerações de SEE et al. (1993), que afirmam que esta complicação pode ser decorrente da perda de gás através dos trocartes.

A hipotrofia observada no testículo posicionado no abdome corresponde aos achados relatados por SWINDLE (1988), que observou infertilidade progressiva em coelhos, a partir de uma semana após a produção de modelo de criptorquidismo bilateral nessa especie.

Uma das etapas fundamentais para o desenvolvimento do cirurgião endoscópico, é o treinamento em animais de experimentação (DENT, 1992; BUESS \& CREUZ, 1993). No presente estudo, o modelo de criptorquidismo em coelhos mostrou-se de 
fácil realização, tendo, esta espécie, permitido a simulação clínica para treinamento diagnóstico e cirúrgico do procedimento de criptorquidectomia laparoscópica realizado em cães e descrito por FREEMAN \& HENDRICKSON (1998). Desta forma, a viabilidade desse modelo em uma espécie animal de laboratório, permite sua utilização em cursos de treinamento de cirurgia endoscópica para cirurgiões veterinários, sem a utilização de espécies domésticas, como o cão.

De forma semelhante à cirurgia de colicistectomia, realizada em suínos em cursos de treinamento laparoscópicos de cirurgiões humanos, como procedimento básico (BUESS \& CREUZ, 1993 e BECK et al., 1996), a criptorquidectomia para o cirurgião veterinário pode também significar procedimento importante para cirurgias endoscópicas, pois além da freqüência elevada com que ocorre nos animais, o criptorquidismo abdominal tem como tratamento de eleição, a remoção cirúrgica do testículo (BOOTHIE, 1998). Outrossim, é importante destacar que a criptorquidectomia laparoscópica cumpre os tempos cirúrgicos laparoscópicos enumerados por BUESS \& CREUZ (1993), como etapas importantes do treinamento cirúrgico na colicistectomia, sendo esses: realização do pneumoperitônio, introdução dos trocartes, dissecação, grampeamento ou ligadura de vasos, liberação intracavitária do órgão, revisão local e da cavidade, exérese e fechamento da parede.

Os tempos decrescentes, registrados na realização da orquiectomia, ressaltam o valor do treinamento na curva de aprendizagem e desenvolvimento da equipe cirúrgica em procedimentos endoscópicos, conforme BECK et al. (1996), FRIEDMAN \& PACE (1996) e FRIED et al. (1999). No presente trabalho, foi possível observar que a dissecação, ligadura, liberação testicular e ensacamento, com remoção do testículo, que correspondem a manobras de habilidade motora na cirurgia endoscópica, demonstraram, igualmente, redução gradativa nos seus tempos de execução. Esses aspectos destacam a repetição na aprendizagem, característica que BUESS \& CREUZ (1993) ressaltaram, afirmando que uma maneira nova de operar necessita e merece, uma maneira nova de aprender e ensinar.

\section{CONCLUSÕES}

Baseado nas condições em que este estudo foi realizado e nos resultados obtidos, podese concluir que:

O coelho presta-se como modelo experimental para estudos de criptorquidismo unilateral, o qual é facilmente exeqüível, simulando de maneira adequada a situação clínica encontrada em animais domésticos de pequeno porte;

a técnica de criptorquidectomia laparoscópica mostrouse efetiva para coelhos, podendo esta espécie ser utilizada como modelo de treinamento laparoscópico para cirurgia veterinária.

\section{REFERÊNCIASBIBLIOGRÁFICAS}

ANDZE, G.O. et al. The role of therapeutic laparoscopy in the surgical treatment intra-abdominal testes in children. Chir Pediatr v.31, n.6, p.299-302, 1990.

BECK, C.A.C. et al. Curso básico de videolaparoscopia experimental: a busca de um modelo didático. In: CONGRESSO BRASILEIRO DE CIRURGIA E ANESTESIOLOGIA VETERINÁRIA, 2., 1996, Ribeirão Preto. Anais... Ribeirão Preto : CBCAV, 1996. p.110-111.

BECKER, J.M. Training and credentialing for endoscopic surgery. In: LOUGHLIN, K.R.; BROOKS, D.C. Principles of endosurgery. Cambridge : Blackwell Science, 1996. Cap.2, p.9-20.

BOOTHE, H.W. Testículos e epididimos. In: SLATTER, D.H. Manual de cirurgia de pequenos animais. São Paulo : Manole, 1998. V.2, cap.97, p.1581-1592.

BRUN, M.V.; BECK, C.A.C. Aplicações clínicas e experimentais da laparoscopia em cães - artigo de revisão. Rev Fac Zoot Vet Agron, PUCRS, v.5-6, n.1, 1998-1999, 1999.

BUESS, G.; CREUZ, O. Treinamento em cirurgia vídeoendoscópica. In: Cirurgia vídeo-endoscópica. Rio de Janeiro : Revinter, 1993. Cap.5, p.53-69.

BURKE, T. J.; REYNOLDS, H.A. The testis. In: BOJRAB, M. J. Pathophysiology in small animal surgery. Philadelphia: Lea \& Febiger, 1983. p 454-459.

DAVIS, E.W. Laparoscopic cryptorchidectomy in standing horses. Vet Surg, v.26, n.4, p.326-331, 1997.

DE LA TORRE, R. A.; SCOTT, J. S.; BLUMENTHAL, M. F. Management of intraabdominal testicle during laparoscopic repair. Surg Endosc, v.9, n.6, p.711-713, 1995.

DENT, T.L. Treinamento, credenciamento e avaliação na cirurgia laparoscópica. Clínicas Cirúrgicas da América do Norte: Laparoscopia para o Cirurgião Geral. v.5, p. 10211030, 1992.

FISCHER, A.T.; VACHON, A.M. Laparoscopic intra-abdominal ligation and removal of cryptorchid testes in horses. Equine Vet J, v.30, n.2, 105-108, 1998.

FREEMAN, L.J. Operating room setup, equipment, and instrumentation. In: FREEMAN, L.J. Veterinary endosurgery. St Louis : Mosby, 1998. Cap.1, p.3-23.

FREEMAN, L.J.; HENDRICKSON, D.A. Minimally invasive surgery of the reproductive system. In: FREEMAN, L.J. Veterinary endosurgery. St Louis : Mosby, 1998. Cap.11, p. 205-225. 
FRIED, G.M. et al. Comparison of laparoscopic performance in vivo with performance measured in a laparoscopic simulator. Surg Endosc, v. 13, p.1077-1081, 1999.

FRIEDMAN, R.L.; PACE, B.W. Resident education in laparoscopic cholecystectomy. Surg Endosc, v.10, n.1, p.26$28,1996$.

FROELING, F.M. et al. The nonpalpable testis and the changing role of laparoscopy. Urology, v.43, n.2, p.222-227, 1994.

GHEILER, E.L.; BARTHOLD, J.S.; GONZÁLEZ, R. Benefits of laparoscopy and the jones technique for the nonpalpable testis. J Urology, v.158, p.1948-1951, 1997.

GILROY, B.A.; ANSON, L.W. Fatal air embolism during anesthesia for laparoscopy in a dog. J Am Vet Med Assoc, v. 190, n. 5, p. 552-554, 1987.

GOMEZ, H.M. et al. Orquiectomia laparoscópica por retenção testicular intrabdominal em cão. Relato de caso. In: CONGRESSO BRASILEIRO DE CIRURGIA E ANESTESIOLOGIA VETERINÁRIA, 2., 1996, Ribeirão Preto. Anais... Ribeirão Preto : CBCAV, 1996. p.80-81.

HENDRICKSON, D.A.; WILSON, D.G. Laparoscopic cryptorchid castration in standing horses. Vet Surg, v.26, n.4, p.335-339, 1997.

KOGAN, B.A.; GUPTA, R.; JUENEMANN, K.P. Fertility in cryptorchidism: Further development of experimental model. J Urology, v.137, p.128-131, 1987.

PENA, S.J. et al. Laparoscopic surgery in a clinical case of seminoma in a cryptorchid dog. Vet Rec, v.142, n.24, p.671672,1998
PRYOR, J.L. et al. Histologic analysis of orchiopexy in a cryptorchid rabbit model. J Urology, v.142, p.413-414, 1989.

QUINN, F.M.J.; CROCKARD, A.D.; BROWN, S. Reversal of degenerative changes in the scrotal testis after orchidopexy in experimental unilateral cryptorchidism. J Pediat Surg, v.26, n.4, p.451-454, 1991 .

REIF, J. S.; BRODEY, R.S. The relationship between cryptorchidism and canine testicular neoplasia. J Am Vet Med Assoc, v.155, p.2005, 1969.

ROMAGNOLI, S.E. Canine cryptorchidism. Vet Clin North Am Small Anim Pract, v.21, n.3, p.533-544, 1991.

SHONO, T.; SUITA, S. The effect of excision of future scrotal skin on testicular descent in neonatal rats: a new experimental model of cryptorchidism. J Pediat Surg, v.30, n.5, p.734$738,1995$.

SEE, W.A.; MONK, T.G.; WELDON, B.C. Complications of laparoscopy - strategies for prevention and treatment. In: CLAYMAN, R.V.; MCDOUGALL, E.M. Laparoscopic urology. St. Louis : Qualitiy Medical, 1993. 450p. p.183-205.

SWINDLE, M. M. Cryptorchidism. In: SWINDLE, M.M.; ADAMS, R.J. Experimental surgery and physiology: induced animal models of human disease. Baltimore : Williams \& Wilkins, 1988. p.67-68.

WILDT, D. E.; KINNEY, G. M.; SEAGER, S. W.J. Laparoscopy for direct observation of internal organs of the domestic cat and dog. Am J Vet Res, v. 38, n. 9, p. 1429-1432, 1977 